\title{
Archéopages
}

Archéopages

Archéologie et société

42 | 04-07/2015

Construire en terre crue

\section{L'utilisation de la terre architecturale à Pascale et Bérange}

Muriel Gandelin

\section{(2) OpenEdition}

1 Journals

Édition électronique

URL : https://journals.openedition.org/archeopages/1137

DOI : 10.4000/archeopages. 1137

ISSN : 2269-9872

Éditeur

INRAP - Institut national de recherches archéologiques préventives

Édition imprimée

Date de publication : 1 mars 2016

Pagination : 24-25

ISSN : $1622-8545$

\section{Référence électronique}

Muriel Gandelin, "L'utilisation de la terre architecturale à Pascale et Bérange », Archéopages [En ligne], 42 | 04-07/2015, mis en ligne le 01 juillet 2017, consulté le 02 juin 2021. URL : http://

journals.openedition.org/archeopages/1137 ; DOI : https://doi.org/10.4000/archeopages.1137

Ce document a été généré automatiquement le 2 juin 2021.

(c) Inrap 


\title{
L'utilisation de la terre architecturale à Pascale et Bérange
}

\author{
Muriel Gandelin
}

Des fouilles menées sur une vaste zone de $16000 \mathrm{~m}^{2}$, située aux lieux-dits Pascale et Bérange, à cheval sur les communes de Saint-Brès et Mudaison ${ }^{1}$ (Hérault), ont révélé des vestiges se rapportant à une occupation du Néolithique final (culture de Fontbouisse) assez dense, située en bordure de cours d'eau. Les niveaux de sol de cette époque ont été détruits par les travaux agricoles et l'essentiel des découvertes consiste en un réseau de structures fossoyées qui marquent la succession d'occupations retranchées [ill.1]. La surface totale ceinturée peut être estimée entre un hectare et demi et deux hectares. Au final, les vestiges mis au jour correspondent à plusieurs enceintes successives, matérialisées au sol par des fossés au développement curviligne dont la forme suggère l'existence de bastions - et par de profondes tranchées de palissades dont certaines aménagent des entrées en pince de crabe. La variété et la nature des vestiges mobiliers découverts sur l'ensemble du site - abondante céramique et industrie lithique en silex, industrie en os ou en cuivre, macro-outillage et rejets alimentaires - évoquent les vestiges habituellement découverts en contexte d'habitat et laissent penser que ces différentes occupations correspondent à autant d'installations villageoises successives. 
1. Plan du site de Pascale et Bérange (Hérault). Entre la moitié et les deux tiers du réseau principal de fossés ont été impactés par l'opération archéologique et le plan du site a pu être complété, dans sa partie orientale, par une prospection électrique et magnétique.

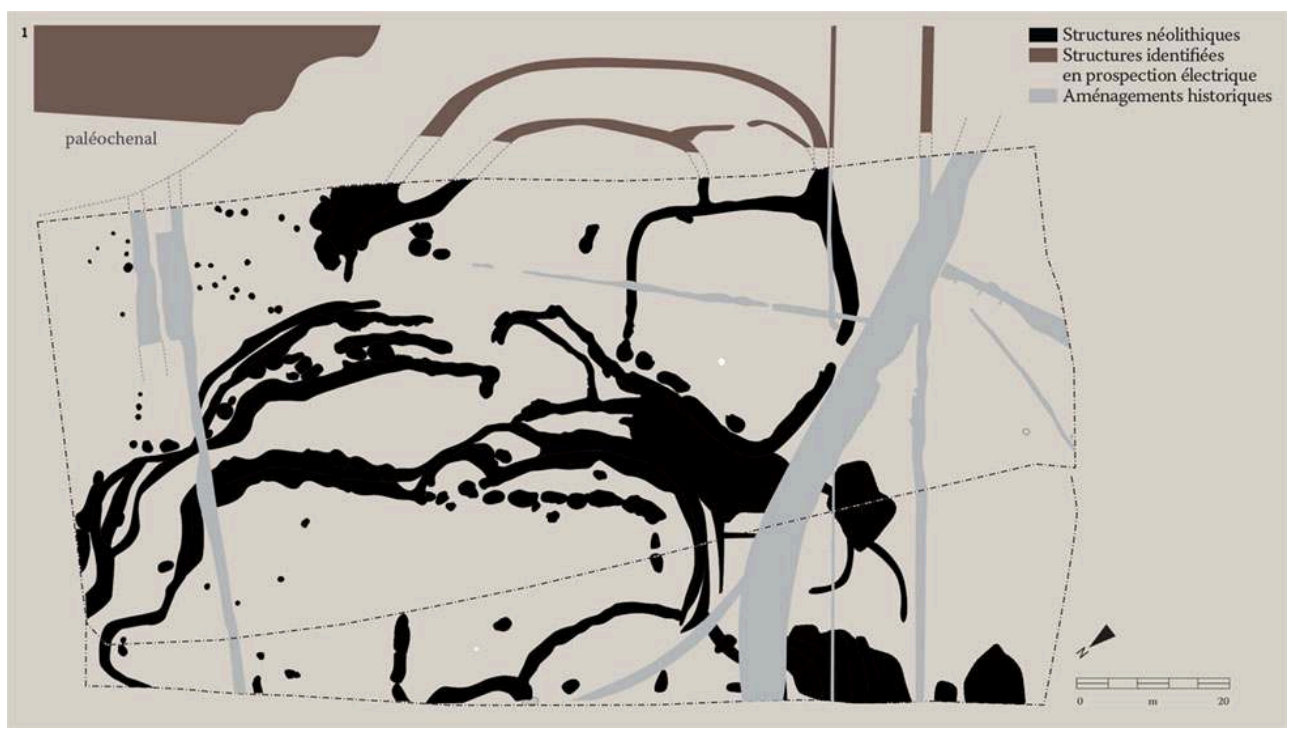

(c) A. Bolo, Inrap

2 Parmi les autres structures découvertes, plusieurs chapelets de creusements allongés, parfois coalescents, semblent suivre le développement des fossés. Ces éléments correspondent à des fosses d'extraction et de préparation de la terre à bâtir destinée à l'édification des élévations des enceintes dont il est difficile de préciser l'architecture. Une partie de la terre utilisée pour ces constructions se trouve remobilisée dans le comblement des fossés, au sein de niveaux pouvant dépasser plusieurs centimètres d'amplitude. Ces vestiges, qui sont les seuls témoins des élévations, se présentent sous plusieurs formes qui peuvent se définir en fonction de leur degré d'ustion. Deux grandes catégories peuvent ainsi être distinguées : la terre crue non chauffée et la terre crue ayant subi l'action du feu à des degrés divers. Dans le cas des éléments ayant subi l'action $\mathrm{du}$ feu, le caractère souvent fortuit de la chauffe explique la variabilité observée dans la couleur, la plasticité et l'état de conservation des différents produits découverts [ill. 2, a.]. La préparation du matériau (l'homogénéisation par malaxage et/ ou la présence naturelle ou l'adjonction volontaire d'éléments non plastiques, minéraux ou organiques, à l'argile) a également une influence directe sur les caractéristiques physico-chimiques des produits obtenus et ces éléments ajoutés peuvent agir comme fondant et/ou comme dégraissant. Dans certains cas, la structure du matériau n'a pas été durablement modifiée par la chauffe ; l'eau interstitielle n'a pas été éliminée des feuillets d'argile : les produits sont fragiles et peuvent reprendre leur plasticité après humidification. Dans d'autre cas, la chauffe a été suffisamment importante pour que l'eau de constitution de l'argile ait été éliminée et que sa structure ait été irrémédiablement détruite : les éléments qui en résultent s'apparentent alors à de la céramique [ill. 2, b.]. Ces fragments accidentellement cuits sont souvent les seuls à conserver des surfaces portant des empreintes interprétables [ill. 2, c.]. Il a ainsi été possible de préciser que la terre était, à Pascale et Bérange, souvent plaquée contre des végétaux souples dont le diamètre excède rarement $1,5 \mathrm{~cm}$. Quelques branches de petit module $(5$ à $10 \mathrm{~cm})$ participaient également à l'armature des constructions. Enfin, en cas de chauffe extrême, les grains de silice présents dans la terre à bâtir ont atteint leur 
point de fusion et le matériau a eu tendance à se vitrifier à des degrés divers, il présente alors un aspect scoriacé, plus ou moins bulleux [ill. 2, d.].

\section{Variété des éléments en terre architecturale.}

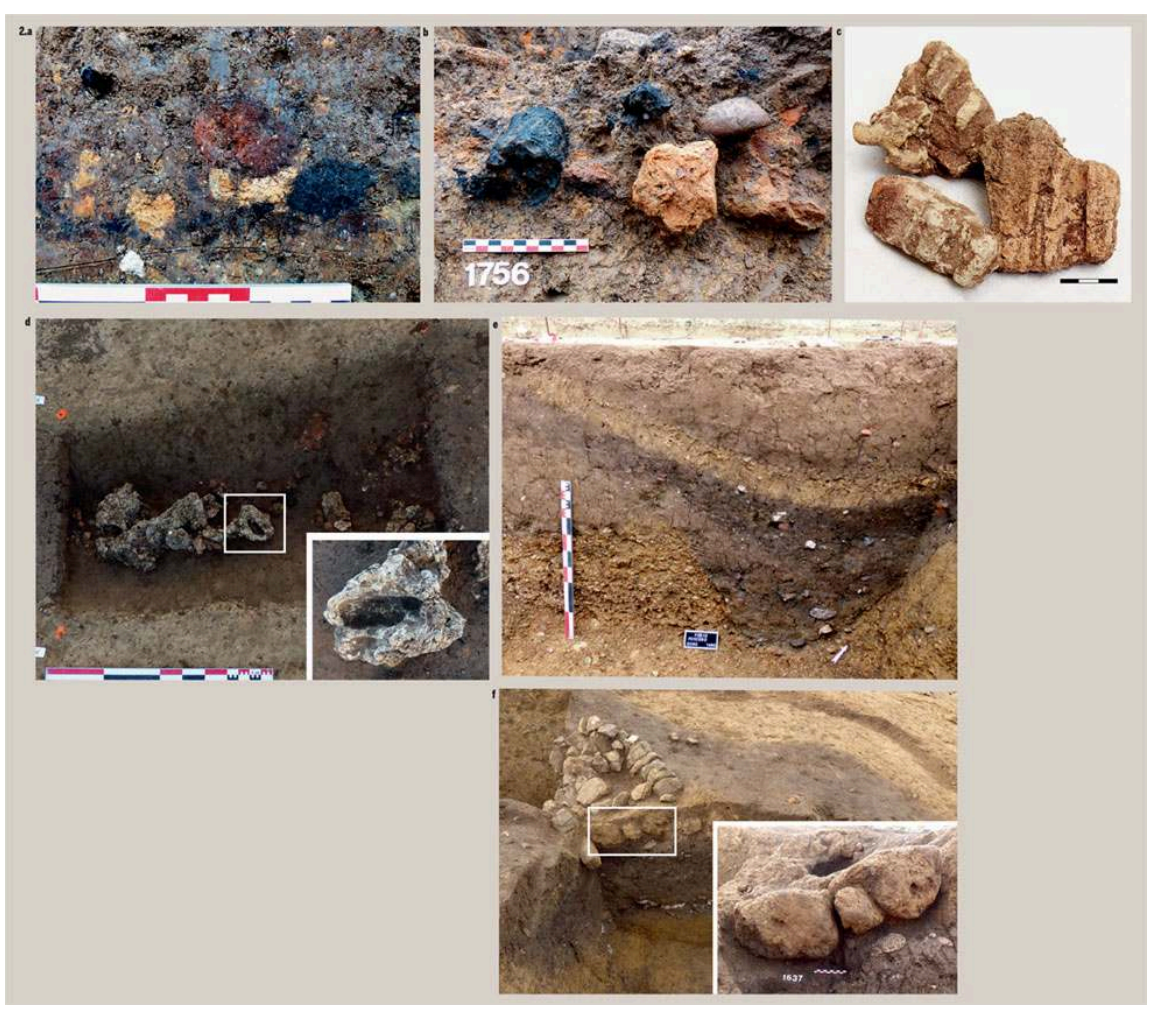

a. et b. terre chauffée ; c. terre cuite portant l'empreinte de végétaux ; d. terre cuite à l'aspect scoriacé ; e. niveau de terre crue en position secondaire dans le comblement d'un fossé ; $f$. base toujours en place d'une construction associant éléments modulaires de terre crue et pierres.

(c) E. Capo, Inrap ; Y. Brossard, Inrap

Dans le cas de la terre à bâtir non chauffée, l'altération subie par le matériau est principalement d'ordre taphonomique et sa conservation est plus aléatoire. La reconnaissance à l'œil nu en est aussi plus difficile. Sur le site de Pascale et Bérange, l'identification de niveaux massifs de terre malaxée, d'une couleur proche du substrat encaissant mais d'une texture fortement homogénéisée, n'a toutefois pas posé de problème [ill. 2, e.]. Dans de rares cas, la forme du matériau initial a pu être retrouvée. Il peut s'agit de pain ou de boule de terre crue de modules variés ; l'utilisation de bauge est également probable mais est plus difficile à identifier.

Une des spécificités du site réside dans la dernière forme architecturale documentée, qui est matérialisée par plusieurs bases de murs. Ces vestiges sont postérieurs au réseau de fossés et palissades et sont installés au sommet de leur comblement. Ils paraissent correspondre aux soubassements massifs et soignés de constructions fortement arasées mais dont le développement semble reprendre, au moins en partie, celui des systèmes fossoyés. Un des éléments les plus remarquables consiste en l'association de la terre et de la pierre dans ces constructions. La terre peut être employée à la façon d'un mortier, pour combler les joints ou bien dans des organisations plus complexes, à l'image de cette base d'architecture constituée d'un premier niveau aménagé par des pains de terre de gros module sur lesquels a été soigneusement installée une assise de pierres [ill. 2, f]. La contemporanéité des deux niveaux semble confortée par la présence d'un 
unique trou de poteau qui traverse la construction sur toute son épaisseur. Cet exemple, remarquablement conservé, reste un témoin exceptionnel de ce type d'architecture mixte.

\section{NOTES}

1. Fouille menée de juillet 2013 à février 2014 sous la direction de Muriel Gandelin, Inrap.

\section{AUTEUR}

MURIEL GANDELIN

Inrap, UMR 5608 « Traces » 